\title{
Genetic Identification and Phylogenetic Analysis of Anaplasma and Ehrlichia Species in Haemaphysalis longicornis Collected from Jeju Island, Korea
}

\author{
Jae Young Oh${ }^{1}$, Bong-Chun Moon ${ }^{3}$, Bo Kyoung Bae' ${ }^{1}$ E-Hyun Shin ${ }^{4}$, Young Hwan Ko ${ }^{5}$, \\ Young-Joo Kim ${ }^{3}$, Yong Ho Park ${ }^{2}$ and Joon-Seok Chae ${ }^{1^{*}}$ \\ ${ }^{1}$ Department of Internal Medicine, Research Institute for Veterinary Science and \\ College of Veterinary Medicine, Seoul National University, Seoul, Korea \\ ${ }^{2}$ Department of Microbiology, College of Veterinary Medicine, Seoul National University, Seoul, Korea \\ ${ }^{3}$ Institute of Environmental Resource Research of Jeju Special Self-Governing Province, Jeju, Korea \\ ${ }^{4}$ Department of Medical Entomology, Korea Center for Disease Control and Prevention, Seoul, Korea \\ ${ }^{5}$ Department of Food Biotechnology, Cheju National University, Jeju, Korea
}

\begin{abstract}
A total of 1,395 Haemaphysalis longicornis ticks collected from Jeju Island of Korea were examined by 16S rRNA gene-based nested PCR for the presence of infection with Anaplasma and Ehrlichia species. Template DNAs to detect the tick-borne pathogens were prepared from a total 506 tick pools. Eight genera of Anaplasma and six Ehrlichia by 16S rRNA gene PCR and sequencing analysis were identified. A. phagocytophilum was the most prevalent (27 [1.9\%]) by nested PCR, followed by A. bovis (5 [0.4\%]), E. chaffeensis (4 [0.2\%]), and A. centrale (1 [0.1\%]). In the phylogenetic analysis based on 16S rRNA sequences, eight genera of Anaplasma group ( $>99.4 \%$ homology) and six Ehrlichia group ( $>99.5 \%$ homology) were close to deposited A. marginale strains (AF309867, AF414874, and FJ226454) and Ehrlichia sp. (DQ324547), respectively. Three Anaplasma species groups A. phagocytophilum (group A), A. bovis (group B), and A. centrale (group C) and one Ehrlichia species E. chaffeensis (group D) were determined by comparing with Anaplasma and Ehrlichia related sequences. First, twenty-eight $A$. phagocytophilum clones belonging to group A were divided into 7 genotypes. The sequence similarity among genotypes A1 to A4 was very high ( $>99.6 \%)$. Genotype B2 was close to $A$. bovis from Korea (99.7\%). Genotype D1 was close to known E. chaffeensis strains (M73222, AF147752, and AY350424) and their similarity value was $99.7 \%$. In conclusion, the genera of Anaplasma/Ehrlichia, A. phagocytophilum, and E. chaffeensis identified in predominant $H$. longicornis ticks were ubiquitous throughout the Jeju Island. The various native groups have been found through sequence identities and phylogenetic analysis.
\end{abstract}

Key Words: Haemaphysalis longicornis, Tick-borne pathogens, Anaplasma phagocytophilum, Ehrlichia chaffeensis

\section{INTRODUCTION}

Anaplasmosis and ehrlichiosis which cause tick-borne

Received: September 10, 2009/ Revised: October 1, 2009

Accepted: October 12, 2009

* Corresponding author: Joon-Seok Chae. Department of Internal Medicine, Research Institute for Veterinary Science and College of Veterinary Medicine, Seoul National University, Seoul 151-742, Korea.

Phone: +82-2-880-1279, Fax: +82-2-880-8662

e-mail: jschae@snu.ac.kr

** This work was supported by National Research Foundation of Korea Grant funded by the Korean Government (2009-0071622). rickettsial diseases in both humans and domestic animals were distributed in the world. Anaplasma genospecies group (A. phagocytophilum, A. bovis, A. platys, A. marginale, and A. centrale) and Ehrlichia genospecies group (E. chaffeensis, E. ewingii, and E. canis) are well known to be pathogenic to human, domestic, and pet animals with acute febrile diseases (1 5). Particularly, A. phagocytophilum and E. chaffeensis, etiologic agents of human granulocytic anaplasmosis (HGA) and human monocytic ehrlichiosis (HME), have been reported mainly from USA and Europe $(6,7)$. In 
Asia, the emergence of intracellular organisms such as Borrelia, Bartonella, Anaplasma, Ehrlichia, and Rickettsia species have been reported frequently from China, Japan, and Korea (8 12). It is known to cause infection or coinfection with only one or mixed infectious agents in ticks and rodents of the various Asian countries. In Korea, A. phagocytophilum and E. chaffeensis were first determined by Heo et al (4) in 2002 by indirect immunofluorescence assay (IFA), Western blotting, and TaqMan real-time PCR from Korean patients. Further studies have been done in Haemaphysalis sp. and Ixodes sp. ticks, dogs, ruminants, and wild animals until the present one (11, 13 15). $H$. longicornis and Ixodes spp. ticks collected from Korea was the most predominant $(>91.8 \%)$ and the prevalence of tick-borne pathogens by TaqMan PCR and species-specific PCR was frequent for some bacteria, including A. platys, $E$. chaffeensis, and Rickettsia sp. in H. longicornis ticks for the most part (10). However, study for bacterial etiologic agents from Jeju Island which is at a distance from Korean inland areas is rare.

Recently, diagnostic methods for detecting of rickettsial pathogens have rapidly changed. Molecular methods that are based on 16S rRNA gene sequence and the nested PCR technique utilizing first PCR product used as template have been reported $(11,15,16)$. However, PCR amplicons by Anaplasma/Ehrlichia associated 16S rRNA gene PCR was not easily amplified with DNAs isolated from ticks and animal.

The aim of this study was to investigate genetic identification and phylogenetic analysis of tick-borne pathogens in H. longicornis ticks collected from Jeju Island of Korea.

\section{MATERIALS AND METHODS}

\section{Survey area and sample collection}

Unfed ticks were collected by dragging and flagging method using $1 \mathrm{~m}^{2}$ flannel flags, in the Jeju Special SelfGoverning Province, Korea. From June 2007 to August 2008, a total of 1,395 ticks were collected from 72 sampling sites into the three survey areas (East, West, and North) which were mostly the grass vegetation, followed by the stock farm, forest, Hallasan National Park, and no livestock (Fig. 1) (Table 1). The tick identification was classified into the various developmental stages according to the standard taxonomic method described by Yamaguti et al (17).

\section{DNA preparation and identification of tick DNA}

After collection, the ticks were placed in bottles containing $70 \%$ ethyl alcohol. The tick samples were disrupted for 5 min with $3.2 \mathrm{~mm}$ stainless steel beads (BioSpec Products, New Haven, UK) in $1.5 \mathrm{ml}$ tubes by TissueLyser II (Universal laboratory mixer-mill disruptor, Qiagen $\mathrm{GmbH}$, Qiagen Strasse Hilden, Germany). Total DNA was isolated from whole tick tissues by using DNeasy Blood \& Tissue kit (Qiagen). The extracted DNA were electrophoresed through $0.8 \%$ agarose gel and the quality of the tick DNA was evaluated by PCR based on the $H$. longicornis tick 5.8S ribosomal DNA spacer (internal transcribed spacer 2, ITS2) with primers HITS2-F (5'-GGTGCTCGAGACTCGTTTTG-3') and HITS2-R (5'-ATTCGCGGTTTACGAGAGAA-3'). Briefly, PCR reaction mixture contained 10 pmol of each primer, $1 \mathrm{U}$ of $\mathrm{HiPi}^{\mathrm{TM}}$ Plus Taq DNA polymerase (ELPIS Biotech, Daejeon, Korea), $20 \mathrm{mM}$ of each dNTP, $50 \mathrm{mM}$ Tris-HCl (pH 9.0), $1.6 \mathrm{mM}\left(\mathrm{NH}_{4}\right)_{2} \mathrm{SO}_{4}, 3.5$ $\mathrm{mM} \mathrm{MgCl}, 0.1 \%$ Tween 20 and $2 \mu$ template tick genomic DNA in a final volume of $25 \mu \mathrm{l}$. The PCR amplification was performed in a PTC-200 thermal cycler (MJ Research, Waltham, MA, USA) with following program: an initial denaturation at $94^{\circ} \mathrm{C}$ for $5 \mathrm{~min}$, followed by 35 cycles consisting of denaturation at $94^{\circ} \mathrm{C}$ for $1 \mathrm{~min}$, annealing at $59^{\circ} \mathrm{C}$ for $1 \mathrm{~min}$, and extension at $72^{\circ} \mathrm{C}$ for $1 \mathrm{~min}$. The PCR products were electrophoresed on a $1.5 \%$ agarose gel, stained with ethidium bromide and visualized under UV light. To confirm the specificity of the tick DNA, total DNAs extracted from the H. flava and Ixodes persulcatus were used as negative controls (Fig. 2).

\section{Detection of Anaplasma and Ehrlichia sp. by PCR}

To detect both the 16S rRNA of Anaplasma and Ehrlichia species, PCR was conducted using primer sets AE1-F (5'-AAGCTTAACACATGCAAGTCGAA-3') and AE1-R (5'-AGTCACTGA CCCAACCTTAAATG-3') in this study. 


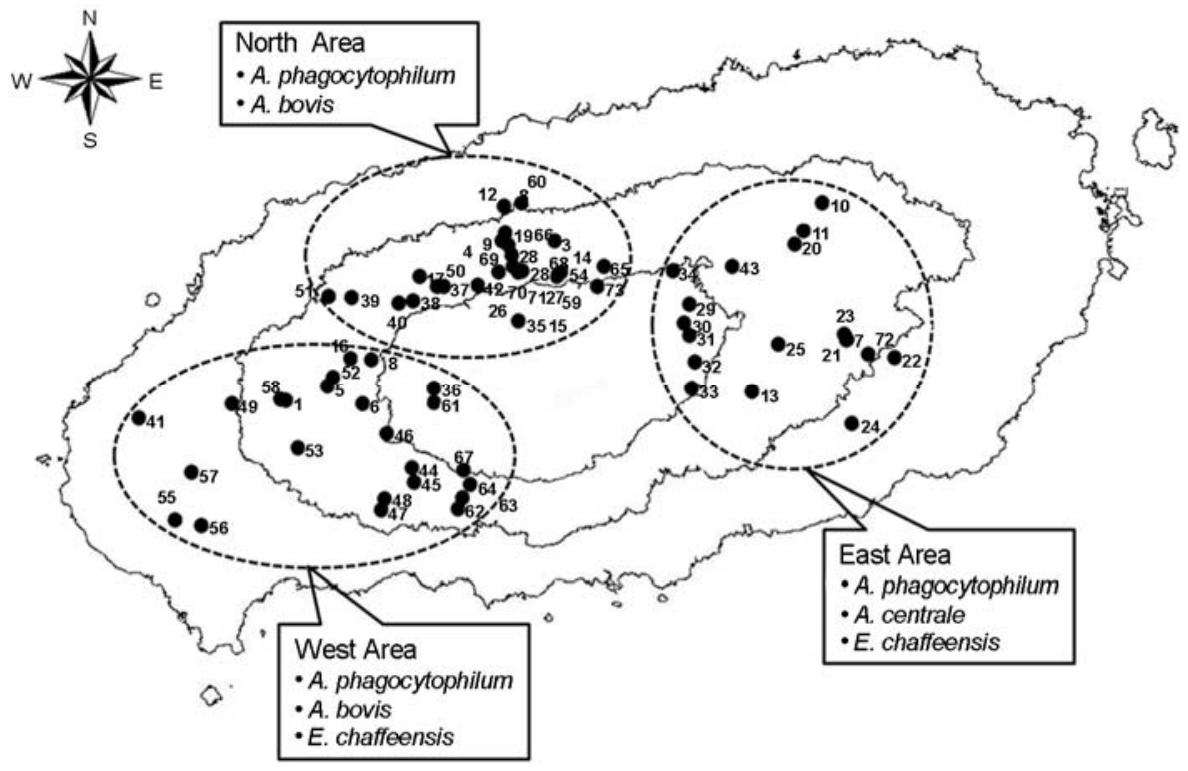

Figure 1. Map of Jeju Island, Korea. Seventy-two tick collection sites indicated by black dots are classified into three areas (East, West, and North) and tick-borne pathogens identified in Jeju Island are indicated by squares.

PCR reaction mixture was performed using conditions mentioned above. Briefly, the PCR amplification was performed with an initial denaturation at $94^{\circ} \mathrm{C}$ for $5 \mathrm{~min}$, followed by 40 cycles consisting of denaturation $94^{\circ} \mathrm{C}$ for $1 \mathrm{~min}, 1 \mathrm{~min}$ of annealing at $59^{\circ} \mathrm{C}$, and 2 min of extension at $72^{\circ} \mathrm{C}$. To determine the prevalence of Anaplasmal Ehrlichia genomic species, nested PCR was performed using $1 \mu \mathrm{l}$ of corresponding primary products (16S rRNA PCR amplicons) as templates. Species-specific primer pairs for PCR were as follows: A. phagocytophilum primers AP-F (5'-GTCGAACGGATTATTCTTTATAGCTTGC-3') and AP-R (5'-CCCTTCCGTTAAGAAGGATCTAATCTCC -3') and E. chaffeensis primers EC-F (5'-CAATTGCTTATAACCTTTTGGTTATAAAT-3') and EC-R (5'-TATAGGTACCGTCATTATCTTCCCTAT-3') according to the method described by Kim et al (10). The annealing for second PCR was performed at $56^{\circ} \mathrm{C}$. The genomic DNA samples extracted from A. phagocytophilum provided by J. Stephen Dumler (Johns Hopkins University School of Medicine, Baltimore, MD, USA) and E. chaffeensis Arkansas strain provided by J. Dawson (CDC, Atlanta, GA, USA) were used as a positive control for detecting tick-borne pathogens Anaplasma and Ehrlichia species, respectively.

\section{Cloning, sequencing analysis, and phylogenetic analysis}

The $\sim 1,406 \mathrm{bp}$ of $16 \mathrm{~S}$ rRNA PCR products, $\sim 926 \mathrm{bp} \mathrm{A}$. phagocytophilum nested PCR products and $\sim 390$ bp E. chaffeensis nested PCR products were eluted from the agarose gel using the Qiagen gel extraction kit (Qiagen), and cloned into the PCR cloning vector pGEM-T easy vector (Promega Co., Madison, WI, USA) and were transformed into competent Escherichia coli JM109 cells; E. coli containing recombinant plasmids were extracted using the SV Minipreps DNA purification system (Promega) and identified by PCR amplification. The sequence analysis of the purified recombinant plasmid DNA was performed with T7 and SP6 promoter primer set using an ABI PRISM 3700 DNA analyzer (Applied Biosystems, Foster City, CA, USA). The sequence data were analyzed using Chromas software (Ver 2.33, http://www.technelysium.com.au/chromas.html) and were aligned using CLUSTAL X (Ver 2.0, http:// www.clustal.org/). Aligned sequences were examined with a similarity matrix. To access the relationships between individual pathogens, the phylogenetic tree was constructed by the UPGMA (unweighted pair group method with arithmetic mean) method with 1,000 bootstrap resamplings using MEGA software (http://www.megasoftware.net/).

\section{Nucleotide sequence accession numbers}

The GenBank accession numbers of 16S rRNA gene sequences and specific genospecies sequences related to Anaplasma/Ehrlichia pathogens for sequence comparisons 


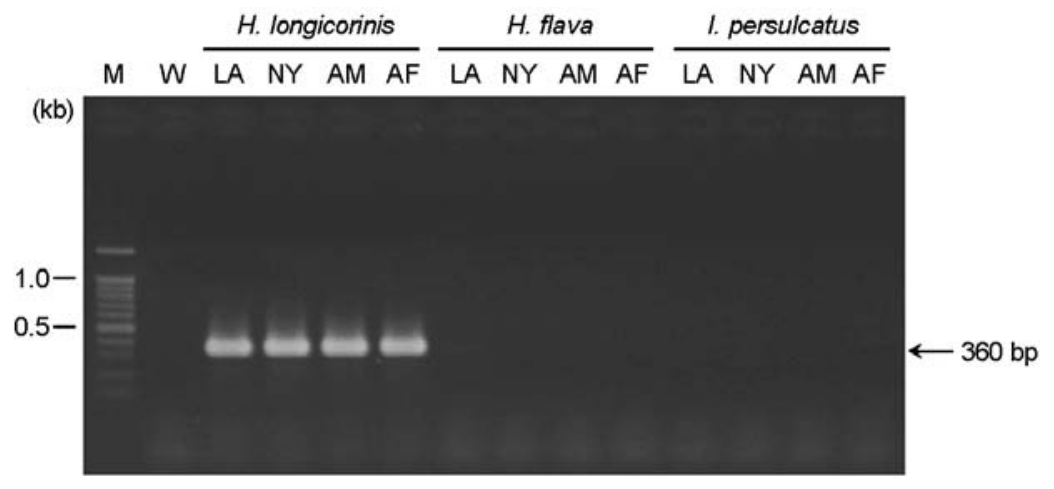

Figure 2. Agarose gel electrophoresis of $H$. longicornis tick 5.8S rRNA internal transcribed spacer 2 gene (360 bp amplicons). Lane M, 100 base pair size marker. Abbreviation of each lane is as follows: $\mathrm{W}$, water control; LA, larva; NY, nymph; AM, adult male; AF, adult female. were as follows; Anaplasma sp. 201-3 from Japan (EU368723), Anaplasma sp. NS108 from Japan (AB454076), Anaplasma sp. from China (FJ69956), A. phagocytophilum from USA (AY055469), A. phagocytophilum from USA (AY055469), A. phagocytophilum from UK (AY082656), A. phagocytophilum from Sweden (AY527213), A. phagocytophilum from Korea (AF470699), A. phagocytophilum from Korea (AF470701), A. platys from Japan (AY077619), A. bovis from Korea (AF470698), A. bovis from Japan (AB196475), A. centrale from USA (AB211164), A. centrale from The Netherlands (AF318944), A. centrale from France (AF283007), A. ovis from China (EF587237), A. ovis from The Netherlands (AF318945), A. marginale from Japan (FJ226454), A. marginale from China (AJ633048), A. marginale from Australia (AF414874), A. marginale from USA (AF309867), Ehrlichia sp. FN147 from Japan (AB196303), Ehrlichia sp. from China (DQ324547), Ehrlichia sp. HF565 from Japan (AB024928), Ehrlichia sp. from Japan (AB028319), E. canis from USA (M73226), E. canis from Spain (AY394465), E. chaffeensis from Korea (AY350424), E. chaffeensis 0214 form Korea (DQ402484), E. chaffeensis from China (AF147752), E. chaffeensis from USA (M73222), Candidatus Ehrlichia shimanensis from Japan (AB074459), E. ewingii from USA (AY093440), and E. ovina from The Netherlands (AF318946) (Fig. 3 5). In the present study, the 22 unique DNA sequences of Anaplasma and Ehrlichia identified from H. longicornis ticks were deposited in GenBank under the accession numbers (GU046563 GU046565, GU064895 GU064903, and GU075695 GU075704) (Fig. 3 5).

\section{RESULTS}

\section{Identification and prevalence of the tick}

All ticks collected from Jeju Island were identified as $H$. longicornis by their morphological patterns. As a result, of the total 1,395 ticks (506 pools) from 72 sampling sites in three survey areas (East, West, and North); nymph was the most frequently collected, which account for 1,043 ticks (74.8\%), followed by 200 female adult ticks (14.3\%), 149 male adult ticks (10.7\%), and 3 larva ticks $(0.2 \%)$. A total of 506 tick pools were first detected by PCR using specific primer sets for amplifying the $360 \mathrm{bp}$ fragment of the $H$. longicornis 5.8S rRNA ITS2 gene and compared with the classified other genus ticks, $H$. flava and I. persulcatus (Fig. 2).

\section{Prevalence and regional distribution of tick-borne pathogens by species-specific PCR}

Both Anaplasma and Ehrlichia 16S rRNA genes associated with genus and genomic species were detected by conventional PCR and species-specific nested PCR assay with $H$. longicornis tick DNAs collected from Jeju Island, Korea. First, we amplified a total of 506 tick pool DNA extracts (total 1,395 ticks) by conventional PCR using $16 \mathrm{~S}$ rRNA primer pairs for genus Anaplasma/Ehrlichia and as a result, fifty-one Anaplasma/Ehrlichia related pathogens were evaluated. Of these pathogens, fourteen 16S rRNA gene PCR amplicons, which were positives for $8(0.6 \%)$ Anaplasma species and 6 (0.4\%) Ehrlichia species by analyzing the sequence alignments based on the GenBank 
Table 1. Prevalence of total ticks (number of pools) and tick-borne pathogens identified from the three main survey areas during 2007 and 2008 in Jeju Island, Korea

\begin{tabular}{|c|c|c|c|c|c|c|c|c|}
\hline \multirow{2}{*}{$\begin{array}{c}\text { Survey } \\
\text { areas } \\
\left(n=72^{a}\right)\end{array}$} & \multirow[b]{2}{*}{ Stages } & \multirow{2}{*}{$\begin{array}{l}\text { No. of ticks } \\
\left(\text { no. of pools }{ }^{b} \text { ) }\right.\end{array}$} & \multicolumn{6}{|c|}{ No. of PCR-positive samples $\left(\%^{c}\right)$} \\
\hline & & & $\begin{array}{l}\text { Anaplasma } \\
\text { spp. }\end{array}$ & $\begin{array}{c}\text { Ehrlichia } \\
\text { spp. }\end{array}$ & A. phagocytophilum & A. bovis & A. centrale & E. chaffeensis \\
\hline \multirow{5}{*}{$\begin{array}{c}\text { East } \\
(n=23)\end{array}$} & Larva & $3(1)$ & 0 & 0 & 0 & 0 & 0 & 0 \\
\hline & Nymph & $342(49)$ & $5(1.5)$ & $1(0.3)$ & $3(0.9)$ & 0 & 0 & $1(0.3)$ \\
\hline & Male & $70(70)$ & $1(1.4)$ & 0 & $2(2.9)$ & 0 & $1(1.4)$ & $1(1.4)$ \\
\hline & Female & $79(79)$ & 0 & 0 & $1(1.3)$ & 0 & 0 & $1(1.3)$ \\
\hline & Subtotal & 494 (199) & $6(1.2)$ & $1(0.2)$ & $6(1.2)$ & 0 & $1(0.2)$ & $3(0.6)$ \\
\hline \multirow{4}{*}{$\begin{array}{l}\text { West } \\
(n=25)\end{array}$} & Nymph & $473(70)$ & $1(0.2)$ & $1(0.2)$ & $6(1.3)$ & 0 & 0 & $1(0.2)$ \\
\hline & Male & $51(51)$ & $1(2.0)$ & 0 & $6(11.8)$ & $1(2.0)$ & 0 & 0 \\
\hline & Female & $69(69)$ & 0 & $1(1.5)$ & $3(4.4)$ & $3(4.4)$ & 0 & 0 \\
\hline & Subtotal & $593(190)$ & $2(0.3)$ & $2(0.3)$ & $15(2.5)$ & $4(0.7)$ & 0 & $1(0.2)$ \\
\hline \multirow{4}{*}{$\begin{array}{l}\text { North } \\
(n=24)\end{array}$} & Nymph & $228(37)$ & 0 & 0 & $3(1.3)$ & 0 & 0 & 0 \\
\hline & Male & $28(28)$ & 0 & 0 & 0 & 0 & 0 & 0 \\
\hline & Female & $52(52)$ & 0 & $3(5.8)$ & $3(5.8)$ & $1(1.9)$ & 0 & 0 \\
\hline & Subtotal & 308 (117) & 0 & $3(1.0)$ & $6(2.0)$ & $1(0.3)$ & 0 & 0 \\
\hline \multirow{5}{*}{ Total } & Larva & $3(1)$ & 0 & 0 & 0 & 0 & 0 & 0 \\
\hline & Nymph & $1,043(156)$ & $6(0.6)$ & $2(0.2)$ & $12(1.4)$ & 0 & 0 & $2(0.2)$ \\
\hline & Male & $149(149)$ & $2(1.4)$ & 0 & $8(5.4)$ & $1(0.7)$ & $1(0.7)$ & $1(0.7)$ \\
\hline & Female & $200(200)$ & 0 & $4(2.0)$ & $7(3.5)$ & $4(2.0)$ & 0 & $1(0.5)$ \\
\hline & Total & $1,395(506)$ & $8(0.6)$ & $6(0.4)$ & 27 ( 1.9$)$ & $5(0.4)$ & $1(0.1)$ & $4(0.2)$ \\
\hline
\end{tabular}

${ }^{a}$ Number of collection sites from the three main survey areas of Jeju Island.

${ }^{b}$ Number of pools for developmental tick stages is as follows, three ticks per pool for larva; five to 7 ticks per pool for nymph; one tick per pool for male and female adults.

${ }^{c}$ PCR-positive pathogens are calculated by minimum infection rate (MIR). MIR = Number of positive pools/total number of ticks tested $\times 100$

database for accession numbers (Table 1). The most commonly isolated pathogen in $H$. longicornis ticks was $A$. phagocytophilum (27 [1.9\%]), followed by A. bovis (5 $[0.4 \%])$, E. chaffeensis (4 [0.2\%]), and A. centrale (1 [0.1\%]) (Table 1). The prevalence of infection with $A$. phagocytophilum according to the minimum infection rate (MIR) from the three survey areas was $15(2.5 \%)$ in the west, followed by the north area $6(2.0 \%)$, and east area 6 (1.2\%). The MIR of A. phagocytophilum according to tick stage was $8(5.4 \%)$ in the male adult ticks, followed by the female adult ticks 7 (3.5\%), and nymph 12 (1.4\%). By survey areas, E. chaffeensis was detected in the east and west area, whereas genera of Anaplasma were detected in all survey areas. Eight Anaplasma positive were $6(0.6 \%)$ nymph pools and $2(1.3 \%)$ male adults and six Ehrlichia positive were $2(0.2 \%)$ nymph pools and $4(2.0 \%)$ female adults, respectively. Twenty-four (1.7\%) tick-borne PCR positive pathogens identified in the west area was the most prevalent, followed by the east area (17 [1.2\%]), and the north area $(10[0.7 \%])$. A. bovis and E. chaffeensis were mostly found in the west $(4[0.3 \%])$ and in the east $(3$ $[0.2 \%])$, respectively.

\section{Phylogenetic analysis of Anaplasma and Ehrlichia species}

To identify the Anaplasma and Ehrlichia species detected in ticks, nucleotide sequences of fourteen PCR amplified products were analyzed and compared with the fragment of 


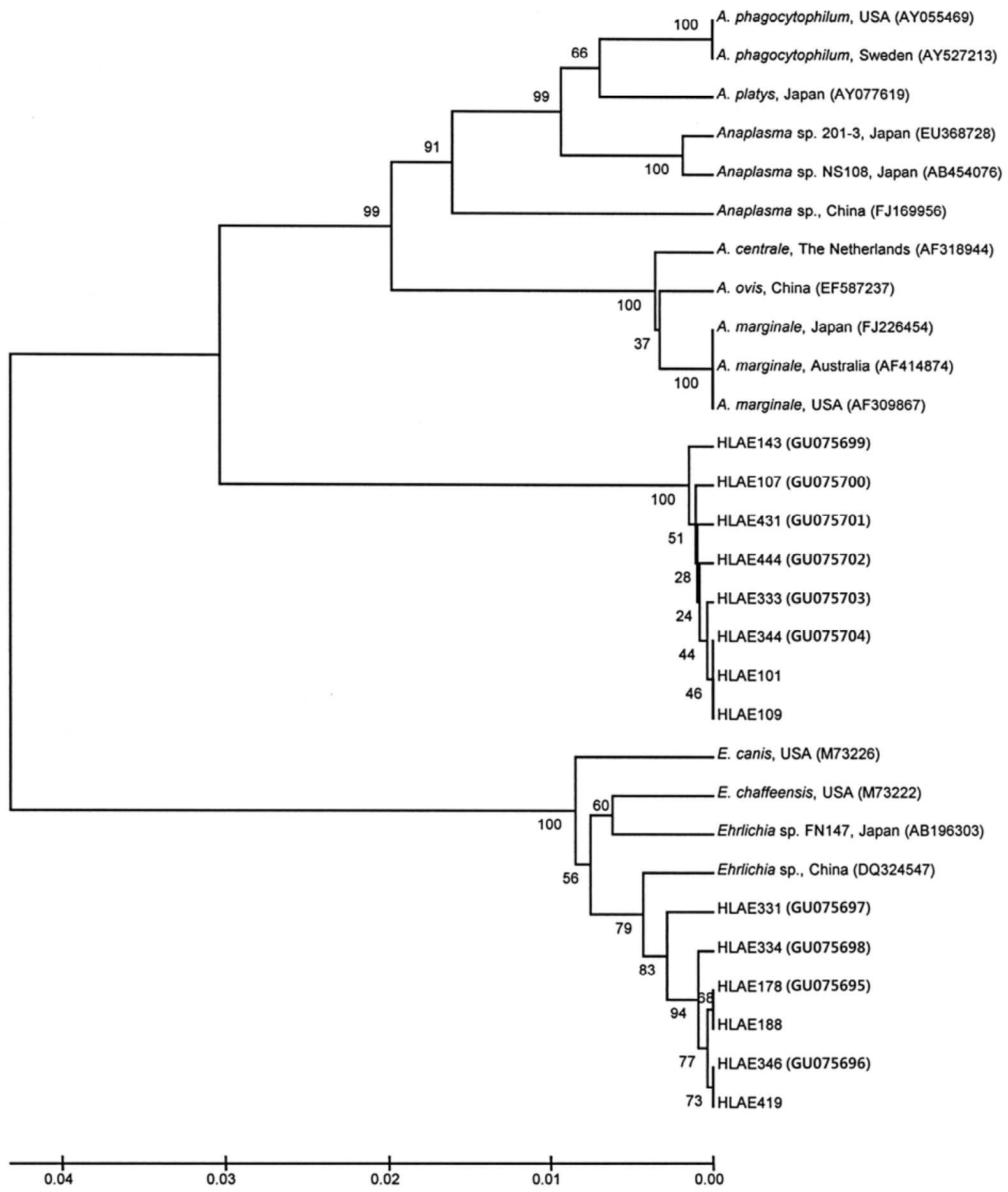

Figure 3. Phylogenetic tree based on 1,406 bp sequences of Anaplasma and Ehrlichia species collected in Jeju Island of Korea. The phylogenetic tree was constructed based on the alignment of 16S rRNA gene sequences by CLUSTAL W and followed by the unweighted pair group method with arithmetic mean (UPGMA) method with 1,000 bootstrap resamplings using MEGA software. The GenBank accession numbers are in parentheses.

16S rRNA sequences and its internal sequences of various Anaplasma and Ehrlichia species obtained from the Gen-
Bank database. Through phylogenetic analysis, the $16 \mathrm{~S}$ rRNA gene sequences were divided into two high homolo- 


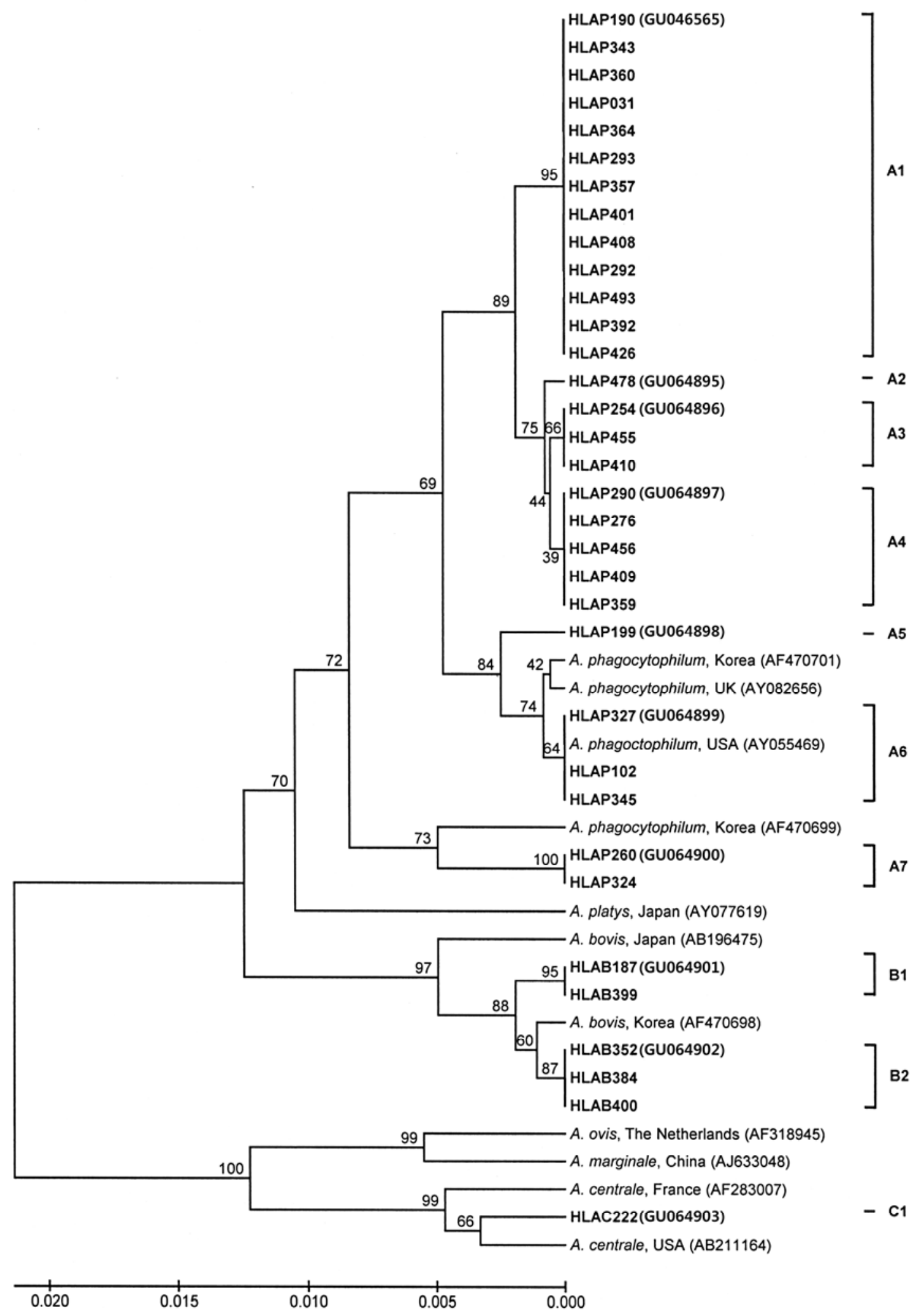

Figure 4. Phylogenetic tree based on 926 bp gene sequence of A. phagocytophilum, A. bovis, and A. centrale. The phylogenetic tree was constructed based on the alignment of Anaplasma gene sequences obtained from species-specific nested PCR assay, by CLUSTAL W and followed by the UPGMA method with 1,000 bootstrap resamplings using MEGA software. 


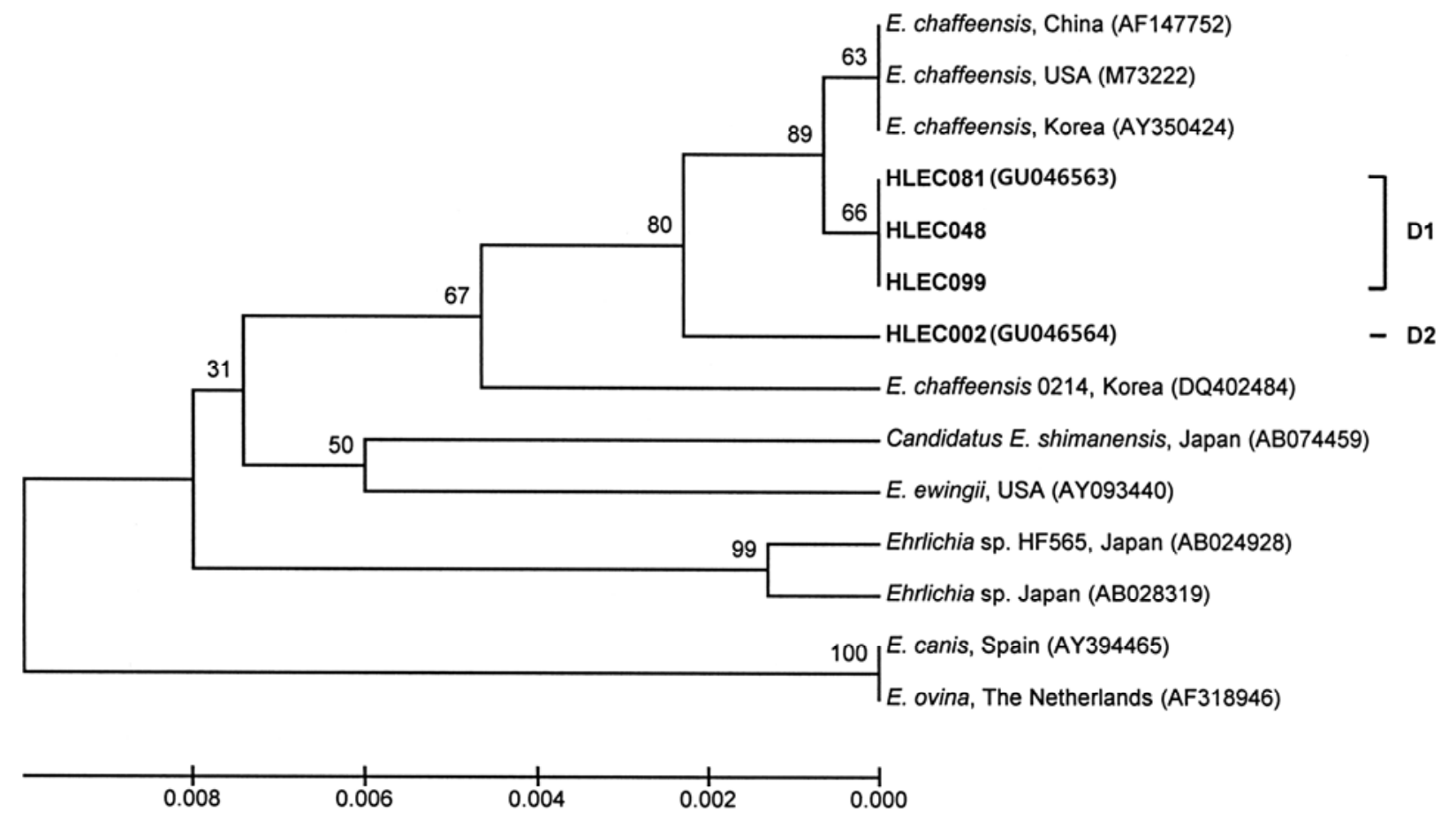

Figure 5. Phylogenetic tree based on 390 bp sequence of E. chaffeensis. The phylogenetic tree was constructed based on the alignment of Ehrlichia gene obtained from species-specific nested PCR assay, by CLUSTAL W and followed by the UPGMA method with 1,000 bootstrap resamplings using MEGA software.

gous groups that were identified as 8 Anaplasma sp. and 6 Ehrlichia sp. by comparing those of other gene sequences of Anaplasma and Ehrlichia species available in GenBank database (Fig. 3). The sequence similarity among 8 Anaplasma group ranged from $99.4 \%$ to $99.7 \%$ and was very similar to A. marginale (FJ226454, AF414874, and AF309867), which ranged from $93.9 \%$ to 94.3 . The similarity level among 6 Ehrlichia group ranged from $99.5 \%$ to $99.9 \%$ and was homologous to Ehrlichia sp. from China (DQ324547) ranged from $98.8 \%$ to $99.0 \%$ (Fig. 3).

Fig. 4 shows that the phylogenetic tree of the 3 Anaplasma species groups, including $28 \mathrm{~A}$. phagocytophilum (group A), 5 A. bovis (group B), and 1 A. centrale (genotype $\mathrm{C}$ ), identified by $A$. phagocytophilum specific PCR amplicons and compared with Anaplasma related species available in GenBank. Group A belongs to the $28 \mathrm{~A}$. phagocytophilum clones which ranged from $97.9 \%$ to $98.4 \%$ and divided into 7 genotypes by the sequence similarity. In case of genotype A6 belonging to the three clones showed identical sequences $(100 \%)$ to known $A$. phagocytophilum originated from USA (AY055469). The homogeneity among four genotypes A1, A2, A3, and A4 was high ( $>$ 99.6\%). Genotype A7 including two clones was the most similar to A. phagocytophilum from Korea (AF470699) with 98.9\% homology. The sequence similarity between the two genotypes (B1 and B2) classifying to A. bovis was $99.4 \%$ and these were highly similar with $99.1 \%$ and $99.7 \%$ similarity to known $A$. bovis sequences from Japan (AB196475) and from Korea (AF470698), respectively. Genotype $\mathrm{C}$ (HLAC222) belonging to one A. centrale showed 99.2\% homology to known A. centrale (AB211164, USA) deposited in GenBank (Fig. 4). The phylogenetic tree among E. chaffeensis clones is shown in Fig. 5. Two genotypes D1 and D2 were identified as partial sequence of E. chaffeensis 16S rRNA gene by sequence comparison. D1 and D2 showed $99.7 \%$ and $99.2 \%$ homology to known E. chaffeensis sequences (AY350424, M73222, and AF147752) from GenBank, respectively (Fig. 5). 


\section{DISCUSSION}

Until now, the most prevalent tick species associated to tick-borne pathogens are Haemaphysalis spp. followed by Ixodes spp. throughout Korea. Especially, H. longicornis tick among Haemaphysalis spp. is known to be the most commonly collected species in previous reports $(10,13)$. The present study revealed one tick species $H$. longicornis in 72 collection sites from Jeju Island despite the emergence of several tick spp. from Korea. It is speculated that the geographical distribution of only one tick species collected is thought to be due to natural environment of forest, river, shrub-undergrowth, and the particular climate different from the inland area of Korea.

The methods for the identification of tick-borne pathogens in ticks have been used by real-time quantitative RT-PCR (TaqMan) or nested PCR. However, 16S rRNA genes from ticks and rodents had not been easily amplified in the previous Korean reports. Previous PCR primers ECC and ECB described by Kim et al (10) were used to amplify primary segment of the 16S rRNA using template DNA of nested PCR for detection of Anaplasma/Ehrlichiarelated genospecies groups. In the present study we could not find exact coincide with the numbers of $16 \mathrm{~S}$ rRNA gene positive compared with the numbers of detected Anaplasma/ Ehrlichia genospecies by nested PCR. Although TaqMan PCR method (10) for detecting tick-borne pathogens revealed a high specificity in screening Anaplasma/Ehrlichia 16S rRNA genes, Kim et al (10) did not yet confirm their sequence analysis in domestic ticks. However, the PCR detection and sequence analysis for genus Anaplasma and Ehrlichia genes in our results were successfully identified with modified primer pairs. The majority of Anaplasma and Ehrlichia 16S rRNA PCR amplicons were detected on gel electrophoresis but several negatives of those of positive amplicons also amplified in the nested PCR for genospecies identification. The difference for these PCR results is probably thought to be either due to a variation in the pathogenrelated DNA quantities because of the size of each tick stage or a variation in the number of tick pools during genomic
DNA preparation. Both Anaplasma and Ehrlichia 16S rRNA sequences analyzed from $H$. longicornis ticks were highly homologous among their groups and were somewhat different compared with those of other countries Japan, China, USA, and Europe. Therefore, genus Anaplasma/ Ehrlichia spp. identified in $H$. longicornis ticks can explain the native tick-borne pathogens in Jeju Island of Korea.

Ticks of genera Haemaphysalis and Ixodes collected from Korea were found to be infected with several tickborne pathogens tested (A. phagocytophilum, A. platys, E. chaffeensis, E. ewingii, and E. canis) $(13,18,19)$. To the best of our knowledge, four tick-borne pathogens $A$. phagocytophilum, A. bovis, A. centrale, and E. chaffeensis were identified in Jeju Island of Korea. Especially, tickborne pathogens infected with A. phagocytophilum were ubiquitous or common among the collection sites and also emerged in all tick stage except for larva, in agreement with previous data that some $A$. phagocytophilum strains were detected in H. longicornis ticks collected in Jeju Island of Korea (13). Thus, $H$. longicornis may be an epidemiologically important vector tick related to $A$. phagocytophilum infection found in this area.

E. chaffeensis, E. canis, and E. ewingii are etiologic agents of canine ehrlichiosis and HME. E. chaffeensis has been mostly detected in $\mathrm{H}$. longicornis ticks collected from the inland areas of Korea, such as Seoul, Gyeonggi province, and Demilitarized zone (DMZ) $(19,20)$. However, E. chaffeensis infection has been rarely reported in human, pet animal and other tick species until recently $(4,15)$. As a result, in contrast to the most predominant A. phagocytophilum genotypes, $E$. chaffeensis pathogens identified from Jeju Island were also not common consistent with previous studies mentioned above.

A. bovis infection is usually associated with some symptoms including fever, lymphadenopathy, depression, and loss of conditioning from the ruminants such as cattle (21). In this study five A. bovis were identified by the nested PCR and sequencing analysis for detection of $A$. phagocytophilum from 506 tick pools. In Korea, one A. bovis-like organism analyzed by $A$. phagocytophilum specific PCR have been reported previously in $H$. longicornis tick 
from Gyeonggi province (20). Recently, A. bovis was first found in the cattle blood of Yonaguni Island, Okinawa, Japan in 2006 (21), since those of the pathogen infected with $A$. bovis detected from deer on the Hokkaido and Honshu Island, Japan, collected during 2001 and 2002 (16). Although it has not provided epidemiologically indisputable evidences and those of pathogen were found in each different local area, ticks pathogens infected might be disseminated by tick-mediated transmission between mammalian hosts or by migration between regions from climate change.

A. bovis are widely distributed in Jeju Island even though they were poor in the present study. Consequently, tickborne pathogens classified into similar or identical genotype groups by the sequence alignment and phylogenetic analysis in this study would be either widespread or infected to human and animals through their life cycle and disease transmission between autochthonous $H$. longicornis ticks infected with these obligate intracellular etiological agents in the nature.

Our results suggest that the genetic diversity of genus Anaplasma/Ehrlichia and their genospecies present in Jeju Island of Korea. Consequently, it is required to compare the epidemiological characterization based on the $16 \mathrm{~S}$ rRNA sequence analysis and phylogenetic analysis of $E$. chaffeensis agents identified in the human and wild animals living in Jeju Island and moreover, it is necessary to clarify the pathogenicity factors of A. phagocytophilum, A. bovis, A. centrale, and E. chaffeensis detected in this study to both human and domestic animals in Korea.

\section{REFERENCES}

1) Dumler JS, Bakken JS. Ehrlichial diseases of humans: emerging tick-borne infection. Clin Infect Dis 1995;20: 1102-10.

2) Murphy GL, Ewing SA, Whitworth LC, Fox JC, Kocan AA. A molecular and serologic survey of Ehrlichia canis, E. chaffeensis, and E. ewingii in dogs and ticks from Oklahoma. Vet Parasitol 1998;79:325-39.

3) Inokuma $H$, Ohno K, Onishi T, Raoult D, Brouqui P.
Detection of ehrlichial infection by PCR in dogs from Yamaguchi and Okinawa Prefectures, Japan. J Vet Med Sci 2001;63:815-7.

4) Heo EJ, Park JH, Koo JR, Park MS, Park MY, Dumler JS, Chae JS. Serologic and molecular detection of Ehrlichia chaffeensis and Anaplasma phagocytophila (human granulocytic ehrlichiosis agent) in Korean patients. J Clin Microbiol 2002;40:3082-5.

5) Kawahara M, Rikihisa Y, Lin Q, Isogai E, Tahara K, Itagaki A, Hiramitsu Y, Tajima T. Novel genetic variants of Anaplasma phagocytophilum, Anaplasma bovis, Anaplasma centrale, and a novel Ehrlichia sp. in wild deer and ticks on two major Islands in Japan. Appl Environ Microbiol 2006;72:1102-9.

6) Bakken JS, Dumler JS. Human granulocytic ehrlichiosis. Clin Infect Dis 2000;31:554-60.

7) Dumler JS, Choi KS, Garcia-Garcia JC, Barat NS, Scorpio DG, Garyu JW, Grab DJ, Bakken JS. Human granulocytic anaplasmosis and Anaplasma phagocytophilum. Emerg Infect Dis 2005;11:1828-34.

8) Uchida T, Yan Y, Kitaoka S. Detection of Rickettsia japonica in Haemaphysalis longicornis ticks by restriction fragment length polymorphism of PCR product. J Clin Microbiol 1995;33:824-8.

9) Shimada Y, Inokuma H, Beppu T, Okuda M, Onishi T. Survey of Ixodid tick species on domestic cats in Japan. Vet Parasitol 2003;111:231-9.

10) Kim CM, Yi YH, Yu DH, Lee MJ, Cho MR, Desai AR, Shringi S, Klein TA, Kim HC, Song JW, Baek LJ, Chong ST, O'Guinn ML, Lee JS, Lee IY, Park JH, Foley J, Chae JS. Tick-borne rickettsial pathogens in ticks and small mammals in Korea. Appl Environ Microbiol 2006;72:5766-76.

11) Sun J, Liu Q, Lu L, Ding G, Guo J, Fu G, Zhang J, Meng F, Wu H, Song X, Ren D, Li D, Guo Y, Wang J, Li G, Liu J, Lin H. Coinfection with four genera of bacteria (Borrelia, Bartonella, Anaplasma, and Ehrlichia) in Haemaphysalis longicornis and Ixodes sinensis ticks from China. Vector Borne Zoonotic Dis 2008;8:791-5.

12) Jilintai, Seino N, Hayakawa D, Suzuki M, Hata H, Kondo S, Matsumoto K, Yokoyama N, Inokuma H. Molecular survey for Anaplasma bovis and Anaplasma phagocytophilum infection in cattle in a pastureland 
where sika deer appear in Hokkaido, Japan. Jpn J Infect Dis 2009;62:73-5.

13) Kim CM, Kim MS, Park MS, Park JH, Chae JS. Identification of Ehrlichia chaffeensis, Anaplasma phagocytophilum, and A. bovis in Haemaphysalis longicornis and Ixodes persulcatus ticks from Korea. Vector Borne Zoonotic Dis 2003;3:17-26.

14) Lee JH, Park HS, Jung KD, Jang WJ, Koh SE, Kang SS, Lee IY, Lee WJ, Kim BJ, Kook YH, Park KH, Lee $\mathrm{SH}$. Identification of the spotted fever group rickettsiae detected from Haemaphysalis longicornis in Korea. Microbiol Immunol 2003;47:301-4.

15) Yu DH, Li YH, Yoon JS, Lee JH, Lee MJ, Yu IJ, Chae JS, Park JH. Ehrlichia chaffeensis infection in dogs in South Korea. Vector Borne Zoonotic Dis 2008;8:355-8.

16) Kawahara M, Rikihisa Y, Lin Q, Isogai E, Tahara K, Itagaki A, Hiramitsu Y, Tajima T. Novel genetic variants of Anaplasma phagocytophilum, Anaplasma bovis, Anaplasma centrale, and a novel Ehrlichia sp. in wild deer and ticks on two major islands in Japan. Appl Environ Microbiol 2006; 72:1102-9.

17) Yamaguti N, Tipton VJ, Keegan H, Toshioka S. Ticks of Japan, Korea, and the Ryukyu islands. Brigham Young Univ Sci Bull Biol Ser 1971;15:1-126.

18) Chae JS, Kim CM, Kim EH, Hur EJ, Klein TA, Kang TK, Lee HC, Song JW. Molecular epidemiological study for tick-borne disease (Ehrlichia and Anaplasma spp.) surveillance at selected U.S. military training sites/installations in Korea. Ann N Y Acad Sci 2003; 990:118-25.

19) Lee SO, Na DK, Kim CM, Li YH, Cho YH, Park JH, Lee JH, Eo SK, Klein TA, Chae JS. Identification and prevalence of Ehrlichia chaffeensis infection in Haemaphysalis longicornis ticks from Korea by PCR, sequencing and phylogenetic analysis based on $16 \mathrm{~S}$ rRNA gene. J Vet Sci 2005;6:151-5.

20) Chae JS, Yu do H, Shringi S, Klein TA, Kim HC, Chong ST, Lee IY, Foley J. Microbial pathogens in ticks, rodents and a shrew in northern Gyeonggi-do near the DMZ, Korea. J Vet Sci 2008;9:285-93.

21) Ooshiro M, Zakimi S, Matsukawa Y, Katagiri Y, Inokuma H. Detection of Anaplasma bovis and Anaplasma phagocytophilum from cattle on Yonaguni Island, Okinawa, Japan. Vet Parasitol 2008;154:360-4. 